\title{
Loss of vacuolar acidity results in iron-sulfur cluster defects and divergent homeostatic responses during aging in Saccharomyces cerevisiae
}

\author{
Kenneth L. Chen - Toby N. Ven • Matthew M. Crane • Matthew L. C. Brunner • \\ Adrian K. Pun • Kathleen L. Helget • Katherine Brower • Dexter E. Chen • Ha Doan • \\ Justin D. Dillard-Telm • Ellen Huynh • Yen-Chi Feng • Zili Yan • Alexandra Golubeva • \\ Roy A. Hsu • Raheem Knight • Jessie Levin • Vesal Mobasher • Michael Muir • \\ Victor Omokehinde • Corey Screws • Esin Tunali • Rachael K. Tran • Luz Valdez • \\ Edward Yang • Scott R. Kennedy • Alan J. Herr • Matt Kaeberlein • Brian M. Wasko (i)
}

Received: 30 December 2019 / Accepted: 14 January 2020 /Published online: 23 January 2020

(C) American Aging Association 2020

\begin{abstract}
The loss of vacuolar/lysosomal acidity is an early event during aging that has been linked to mitochondrial dysfunction. However, it is unclear how loss of vacuolar acidity results in age-related dysfunction. Through unbiased genetic screens, we determined that increased iron uptake can suppress the mitochondrial respiratory deficiency phenotype of yeast vma mutants, which have lost vacuolar acidity due to genetic disruption of the vacuolar ATPase proton pump. Yeast vma mutants exhibited nuclear localization of Aft1, which turns on the iron regulon in response to iron-sulfur cluster (ISC) deficiency. This led us to find that loss of
\end{abstract}

Electronic supplementary material The online version of this article (https://doi.org/10.1007/s11357-020-00159-3) contains supplementary material, which is available to authorized users.

K. L. Chen · T. N. Ven • M. M. Crane $\cdot$ M. L. C. Brunner •

A. K. Pun - K. Brower - D. E. Chen - H. Doan •

J. D. Dillard-Telm • E. Huynh · Y.-C. Feng · Z. Yan •

A. Golubeva · R. A. Hsu • R. Knight · J. Levin •

V. Mobasher $\cdot$ M. Muir $\cdot$ V. Omokehinde $\cdot$ C. Screws $\cdot$

E. Tunali $\cdot$ R. K. Tran $\cdot$ L. Valdez $\cdot$ E. Yang $\cdot$

S. R. Kennedy · A. J. Herr • M. Kaeberlein

Department of Pathology, University of Washington, Seattle, WA 98195, USA

K. L. Helget • B. M. Wasko $(\bowtie)$

Department of Biology and Biotechnology, University of

Houston-Clear Lake, Houston, TX 77058, USA

e-mail:wasko@uhcl.edu vacuolar acidity with age in wild-type yeast causes ISC defects and a DNA damage response. Using microfluidics to investigate aging at the single-cell level, we observe grossly divergent trajectories of iron homeostasis within an isogenic and environmentally homogeneous population. One subpopulation of cells fails to mount the expected compensatory iron regulon gene expression program, and suffers progressively severe ISC deficiency with little to no activation of the iron regulon. In contrast, other cells show robust iron regulon activity with limited ISC deficiency, which allows extended passage and survival through a period of genomic instability during aging. These divergent trajectories suggest that iron regulation and ISC homeostasis represent a possible target for aging interventions.

Keywords Aging · Geroscience · Iron-sulfur clusters · Vacuolar acidity $\cdot$ Lysosomal acidity Yeast replicative lifespan · DNA damage

\section{Introduction}

Many studies of biological aging are performed with only a few age points. While these types of studies have revealed a multitude of biological processes that become dysfunctional with age, they are unable to reveal the sequence, kinetics, and penetrance of age-associated 
changes. Defining these parameters is necessary in order to better understand the network of failures that results in age-associated pathological physiology. In the budding yeast (Saccharomyces cerevisiae), most aging studies have also been performed on populations of cells, lacking the resolution to identify differences within individual cells. Recent developments in microfluidic device technology have allowed for the characterization of yeast replicative aging with whole-lifespan breadth and single-cell resolution (Chen et al. 2017; Crane and Kaeberlein 2018; Chen et al. 2019). This can allow for the identification of early drivers of aging as well as an understanding of the kinetics and penetrance of these changes, and information about their correlations with ultimate lifespan at single-cell granularity. This information may yield richer insight needed for the development of early interventions to combat age-associated diseases.

The lysosome/vacuole is a central node of cellular metabolism, with critical roles in nutrient sensing and storage, metal homeostasis, organelle maintenance, and protein degradation ( $\mathrm{Li}$ and Kane 2009). Accordingly, lysosomal dysfunction underlies many clinically significant genetic and degenerative diseases (Carmona-Gutierrez et al. 2016; Perera and Zoncu 2016). The lysosome/vacuole is maintained at an acidic $\mathrm{pH}$, which is necessary for proper organelle function. Recent work has shown that the loss of lysosomal/vacuolar acidity is an evolutionarily conserved early life driver of aging and mitochondrial dysfunction (Baxi et al. 2017; Ghavidel et al. 2018; Hughes and Gottschling 2012), and interventions that increase vacuolar acidity have been found to extend lifespan in yeast (Sasikumar et al. 2019).

The acidic environment of the lysosomal/vacuolar lumen is generated by a highly conserved multisubunit proton pump, the vacuolar ATPase (V-ATPase). In mammals, genetic ablation of the V-ATPase function is lethal (Inoue et al. 1999). However, in the budding yeast Saccharomyces cerevisiae, deletion of vacuolar ATPase subunits or dedicated assembly factors ( $v m a$ mutants) is tolerated but results in a variety of physiological dysfunctions. In addition to alkalization of the vacuole, vma mutants exhibit respiratory incompetence (Eide et al. 1993); substantially reduced replicative lifespan (McCormick et al. 2015; Schleit et al. 2013); as well as sensitivity to elevated calcium levels (Ohya et al. 1986), alkaline pH (Serrano et al. 2004), iron depletion
(Davis-Kaplan et al. 2004), reactive oxygen species (Milgrom et al. 2007), and DNA damage (Liao et al. 2007). How loss of V-ATPase activity and the resulting alterations in cellular $\mathrm{pH}$ homeostasis cause these pleiotropic phenotypes and limit lifespan remains unclear.

In both yeast and metazoans, lysosomal activity is necessary for the maintenance of iron homeostasis (Kidane et al. 2006; Milgrom et al. 2007). Iron is essential for many cellular functions and exists in various forms in the cell, including diiron, heme, and ironsulfur clusters (ISCs). In yeast, the iron regulon is a coordinated gene expression program that is activated by iron deficiency and results in increased iron uptake and redistributed iron usage (Philpott and Smith 2013). Interestingly, this program is triggered not by low total iron levels in the cell, but by reduced iron-sulfur cluster (ISC) availability (Chen et al. 2004). ISCs are ancient protein prosthetic groups used in a wealth of essential cellular processes including DNA replication and repair, amino acid production, protein translation, and the electron transport chain (Lill et al. 2012). ISC production is dependent on a series of mitochondrial iron-transfer steps (Lill et al. 2012), and it has been speculated that they are the ultimate reason for the persistence of mitochondria in anaerobic eukaryotes. Indeed, mutations in proteins that utilize or assemble iron-sulfur clusters underlie a plethora of devastating genetic cancer syndromes, hematopoietic abnormalities, and neurological diseases (Lill et al. 2012). Additionally, during conditions of ISC deficiency, genome maintenance is impaired (de la Díaz et al. 2011; Pijuan et al. 2015; Veatch et al. 2009), mimicking a critical evolutionarily conserved hallmark of aging (López-Otín et al. 2013).

We hypothesized that determining how vacuolar acidity results in the pleiotropic defects and respiratory incompetence of $v m a$ mutants would provide insight into how age-associated loss of vacuolar acidity impacts cellular function during aging. Our results indicate that disruption of iron homeostasis is an important hub tying perturbed $\mathrm{pH}$ homeostasis and mitochondrial function. Stemming from this observation, we find that wild-type yeast display ISC defects during aging. Interestingly, we observe a divergence in iron-related homeostatic trajectories that cells can undergo during aging. A large subpopulation of cells appears unable to respond to the ISC-related crisis, while other cells mount the expected compensatory gene expression program that allows these cells to survive genomic instability and achieve a full lifespan potential. 


\section{Methods}

Reagents

Yeast strains used in this study are haploid and derived from the BY4741/4742 (S288C derived) background and genotypes are shown in Table S1. YEP media consisted of 2\% Bacto Yeast Extract (Difco), 1\% Bacto Peptone (Difco), and 2\% glucose (YPD, fermentative conditions) or $3 \%$ glycerol (YPG, respiratory conditions).

\section{Yeast multicopy suppression screen}

The multicopy suppression screen was performed using a YEP13 library ( $2 \mu$, LEU2 vector) containing 5-20 kb inserts of yeast genomic DNA (Nasmyth and Reed 1980). The YEP13 pooled library was transformed into

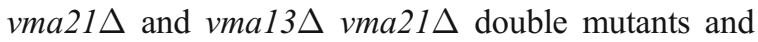
plated onto media lacking leucine to select for plasmid transformation. Approximately 10,000 colonies were then replica plated onto YPG media. For colonies that grew, the plasmid was isolated from yeast using a Qiagen plasmid prep kit (with glass beads added to lysis buffer) and the plasmid was transformed into E. coli, isolated (Qiagen) and sequenced with primers flanking the insertion site to identify the genomic region contained on the plasmid. For validation, FET4 was cloned with $\sim 400 \mathrm{bp}$ of the upstream promoter into the pAG425 (resulting plasmid named pBMW182a) backbone using the Yeast Gateway System Vectors (obtained from Addgene) (Alberti et al. 2007).

Yeast spontaneous and UV-mediated suppression screen

For the spontaneous genetic suppressor screen, vma21s cells were grown to log phase and then plated onto YPG plates. For the UV-mediated suppression screen, vma21 $\Delta$ cells were exposed to UV radiation in an amount empirically determined to kill $\sim 50 \%$ of cells using a UV Stratalinker, and cells were plated on YPG media. Clones that were identified as suppressors were validated by rechecking growth on YPG. To identify the causative suppressing mutation, one strain was whole genome sequenced as described below, and subsequently other strains were Sanger sequenced at the ROXI locus.
Whole genome sequencing

We used pooled linkage analysis to identify the mutation underlying the suppressor mutation (Birkeland et al. 2010). We first crossed the haploid mutant (BW1151) to a wild-type haploid. We then dissected tetrads from this strain and identified suppressor and non-suppressor spore clones based on their growth phenotypes on YPG. We grew overnight cultures from 10 of each class of segregant and counted the cells using a hemocytometer. We then combined $10^{8}$ cells from each culture into two pools corresponding to the two genotypes. We purified genomic DNA from the pooled cells using a QuickDNA Fungal/Bacterial Miniprep kit (Zymo Research). We performed whole genome sequencing of the two pools essentially as described (Lee et al. 2019), except that we used a variant frequency cut-off of 0.8 to identify SNPs. There was a single SNP in the suppressor pool, which was present in 20 out of 20 reads and completely absent from the non-suppressed pool. The mutation maps to the ROXI locus on chromosome 16 and causes an A to T substitution in the first position of the fourth codon (AAA), creating a premature TAA stop codon (roxl-A10T).

Yeast growth assays

Yeast growth assays on solid agar containing media were performed similarly to as previously described (Wasko et al. 2009). Briefly, yeast strains were transferred to into water and diluted to identical optical densities (OD600). Cells were aliquoted into 96 well microplates, fivefold serially diluted in water, and $4.5 \mu \mathrm{l}$ of each sample was pipetted onto the indicated media type using a multichannel pipette. After drying, plates were incubated at $30^{\circ} \mathrm{C}$ for $2-5$ days.

Aconitase activity assay

Aconitase activity assay was based on a previously described method (Tong and Rouault 2006). Briefly, cell extracts were incubated with $0.2 \mathrm{mM}$ phenazine methosulfate, 0.5 mM MTT (3-(4,5-dimethylthiazol-2yl)-2,5-diphenyltetrazolium bromide), $0.25 \mathrm{mM}$ NADP, $2.5 \mathrm{mM}$ cis-aconitic acid, and $0.4 \mathrm{U} / \mathrm{ml}$ isocitrate dehydrogenase and absorbance at $450 \mathrm{~nm}$ was quantified using a plate reader. An isocitrate standard was used to generate a standard curve and experimental data was collected with absorbance values that fell within the 
range of the linear standard curve. The BCA assay was used to determine equal protein amounts to add from each sample.

\section{Flow cytometry}

Cells were grown to log phase in the indicated condition and cells were spun down, transferred into water, and stored on ice briefly until flow cytometry was performed with a FACSCanto II flow cytometer at the University of Washington Pathology Flow Cytometry Core Facility. Excitation was using a 488-nm laser and emission was monitored with a 502 long pass filter and a 530/30 filter. Three biological replicates were measured, each of which consisted of 20,000 cells per condition.

\section{Standard fluorescent microscopy}

Wild-type or vma21 $\Delta$ cells expressing Aft1-GFP were grown at $30{ }^{\circ} \mathrm{C}$ to $\log$ phase in liquid YPD and then transferred to YPD in the presence or absence of $100 \mu \mathrm{g} / \mathrm{ml}$ bathophenanthrolinedisulfonic acid (BPS) or $1 \mathrm{mM}$ iron ammonium sulfate for $2 \mathrm{~h}$ at $30^{\circ} \mathrm{C}$. Cells were then transferred to synthetic media (to reduce background fluorescence) with $2 \%$ glucose with or without $100 \mu \mathrm{g} / \mathrm{ml}$ BPS or $1 \mathrm{mM}$ iron ammonium sulfate and imaged promptly after the media was changed.

\section{Replicative lifespan}

Yeast replicative lifespan and statistical analysis were performed similar to as previously described (Wasko et al. 2013). Cells were patched to fresh YPD plates containing indicated compounds and grown overnight at $30^{\circ} \mathrm{C}$. Virgin daughter cells of $20-40$ cells per condition were selected for replicative lifespan microdissection analysis.

\section{Microfluidics and fluorescence microscopy}

Cells were imaged using a PDMS microfluidic flow chamber with cell traps modified to increase mother cell retention and introduction of multiple chambers to isolate cells of different genotypes in identical environments. Cells were loaded according to previously published methods (Crane et al. 2014). A volumetric flow rate of $1-12 \mu 1 / \mathrm{min}$ per chamber was used. Flow rate was initialized at a low rate to prevent ejection of smaller young mother cells from traps and increased throughout the experiment to reduce cell clogging and to maintain the trapping of large older mother cells. Cells were imaged using a Nikon Ti-2000 microscope with a 40X oil immersion objective, 1.3 NA using the Nikon Perfect Focus System. An enclosed incubation chamber was used to maintain a stable environment at $30{ }^{\circ} \mathrm{C}$. An LED illumination system (Excelitas 110-LED) was used to provide consistent excitation energies, with illumination triggered by camera shutter to prevent excess exposure. Images were acquired using a Hammamatsu Orca Flash 4.0 V2. The camera and stage were controlled by in-house software written in Matlab ${ }^{\circledR}$ and Micromanager. Images were corrected for illumination artifacts. To correct for single pixel biases, 1000 images were acquired with no illumination, and individual pixel means were determined. To correct for flatness of field, fluorescent dye was added to a microfluidic device. One thousand images were acquired each with a small offset in the $\mathrm{x}$ and $\mathrm{y}$ positions (to compensate for microfluidic trap features). Images were dilated, and the median value at each location was used. For every image, pixel-level bias was subtracted and values were multiplied by a flatness of field correction factor. Images were acquired at $5 \mathrm{~min}$ intervals for bright-field. Fluorescence imaging was acquired at $30 \mathrm{~min}$ or $1 \mathrm{~h}$ intervals. For bright-field imaging, three z-sections were $3.5 \mu \mathrm{m}$ intervals. For the fluorescence, 3-7 z-sections were acquired. GFP images were acquired using a Chroma ET49002 filter set. mRuby2 and mCherry images were acquired using a Chroma ET49306 filter set. For pHluorin2 ratiometric imaging, the Chroma GFP ET49002 filter set and a custom ET405/40X excitation and ET525/50 m emission filter set (Chroma) were used. Following data acquisition, cells were segmented and tracked using previously published software (Bakker et al. 2018). Divisions were scored by eye, and errors in cell segmentation and tracking were corrected manually. For Fit2 mean fluorescence, values of maximum projection were interpreted to reflect total protein levels. For vacuolar acidity, for each cell the brightness of the two fluorescence channels were equalized and summed, and the brightest $5 \%$ of pixels were used. The mean of the pixel-level ratio of these channels was used as a measure of vacuolar acidity. For Rad52 foci presence, the value of the brightest 9-pixel square divided by the brightest $2.5 \%$ of the cell was used. A threshold was determined by visual inspection of foci-containing and foci-free cells (1.1 for GFP, 1.05 for mCherry). For Rps2 nuclear 
retention, the value of the brightest 9 pixel square divided by the mean brightness of the cell was used.

Cells were inoculated into SC media (Sunrise Biosciences) with $2 \%$ dextrose and grown overnight $(\sim 12-$ $24 \mathrm{~h}$ ) until log phase. BSA was added immediately prior to loading to prevent adherence to PDMS. During experiments, SC media with $2 \%$ dextrose was used, and cells were imaged for $60-80 \mathrm{~h}$.

\section{Results}

Iron rescues pleiotropic phenotypes following disrupted V-ATPase function

Previous studies suggested that the loss of vacuolar acidity limits lifespan through mitochondrial dysfunction (Hughes and Gottschling 2012). In order to identify links between vacuolar acidity and mitochondrial function, we performed a multicopy screen for suppressors of the mitochondrial respiratory deficiency phenotype in vma mutants. We found that overexpression of the FET4 gene encoding for a low-affinity iron transporter allowed vma21 $\Delta$ cells to grow under respiratory conditions (Fig. 1a). An additional screen for spontaneous and UV-mediated suppression of the vma21 $\Delta$ respiratory deficiency yielded numerous loss of function alleles in the $R O X 1$ gene (Fig. $1 \mathrm{~b}$ and Table S2). Another genetic screen for suppressors of vma21 $\Delta$ sensitivity to an iron chelator also yielded loss of function $R O X 1$ alleles (Table S3). Rox $1 p$ is a transcriptional repressor of genes involved in the yeast hypoxic response, so loss of $R O X 1$ results in activation of yeast hypoxic responsive genes. FET4 is one of the most upregulated targets in roxl $\Delta$ mutants (Jensen and Culotta 2002; Ter Linde and Steensma 2002; Waters and Eide 2002), and FET4 was required for suppression of $v$ ma21 1 respiratory deficiency by mutation of $R O X 1$ (Fig. 1c).

Since Fet4 is an iron importer, we also tested supplementation of the growth media with iron, which also resulted in suppression of the vma21 $\Delta$ respiratory deficiency (Fig. 1d). This iron-mediated rescue of $v m a$ respiratory growth occurred with different forms of iron (II or III) and in multiple vma mutants lacking either cytosolic $\left(\mathrm{V}_{1}\right)$ or membrane associated $\left(\mathrm{V}_{0}\right)$ subunits of the V-ATPase (Fig. S1a). Interestingly, iron supplementation additionally rescued other pleiotropic phenotypes of vma21 $\Delta$ mutants including sensitivity to elevated $\mathrm{pH}$ and calcium, manganese, and oxidative stress induced by paraquat (Fig. 1d). Iron supplementation also rescued respiratory defects in wild-type yeast when the VATPase is chemically inhibited by bafilomycin (Fig. 1e), suggesting that both acute and chronic inhibition of the V-ATPase impairs mitochondrial function by altering iron homeostasis. The short replicative lifespan of vma21 $\Delta$ and vma13 $\Delta$ mutants was additionally rescued by addition of iron and/or sodium ascorbate (Fig. 1f and Fig. S1b). Sodium ascorbate is an antioxidant that can reduce iron from the ferric $\left(\mathrm{Fe}^{3+}\right)$ to ferrous $\left(\mathrm{Fe}^{2+}\right)$ form (de Silva et al. 1997). The addition of sodium ascorbate diminished the iron regulon activation in vma21 1 mutants (Fig. S1c), rescued the sensitivity of vma21 $\Delta$ mutants to an iron chelator, and appeared to qualitatively increase the growth rate of wild-type yeast in the presence of an iron chelator (Fig. S1d). This ability of sodium ascorbate to impact iron homeostasis may occur in three ways. Conceivably, ascorbate may have beneficial effects by reducing iron in the media (making it more soluble and bioavailable), by reducing iron intracellularly, and/or by acting directly as an intracellular antioxidant.

Reduced V-ATPase function results in iron dyshomeostasis

In yeast, the response to low intracellular iron levels is mediated by nuclear localization of the transcription factor Aft1, which activates the iron regulon, transcription of a suite of genes involved in iron assimilation (e.g., FET3, FTR1, and FIT2). Using an Aft1 responsive transcriptional reporter expressing GFP under the control of the FIT2 promoter (Diab and Kane 2013), vma21 $\Delta$ mutants displayed increased levels of GFP fluorescence compared to wild type, which was reduced by the addition of iron (Fig. 2a). There is also nuclear localization of Aft1-GFP in vma21 $\Delta$ cells, and exogenous iron reduced the nuclear localization of GFPtagged Aft1 in vma21 $\Delta$ cells (Fig. 2b). Although $v m a 21 \Delta$ cells clearly induce the iron starvation response, we failed to detect any significant change in total intracellular iron levels (Fig. 2c), which is consistent with prior reports for other vma mutants (Diab and Kane 2013; Szczypka et al. 1997).

Nuclear localization of Aft1 and activation of the iron regulon are triggered by deficits in iron-sulfur cluster availability (Rutherford et al. 2005), suggesting the possibility that ISCs become limiting in $v m a$ cells. Aconitase is a mitochondrial ISC containing 

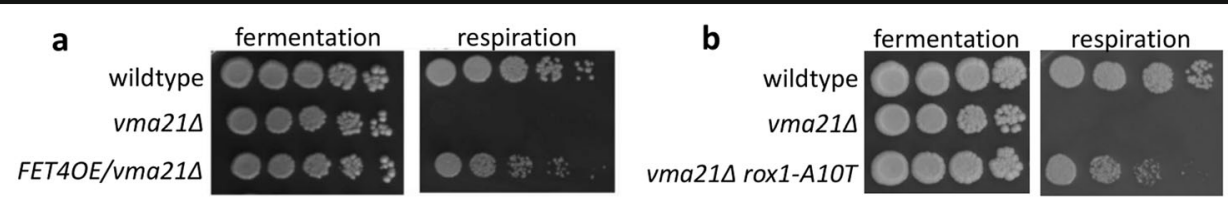
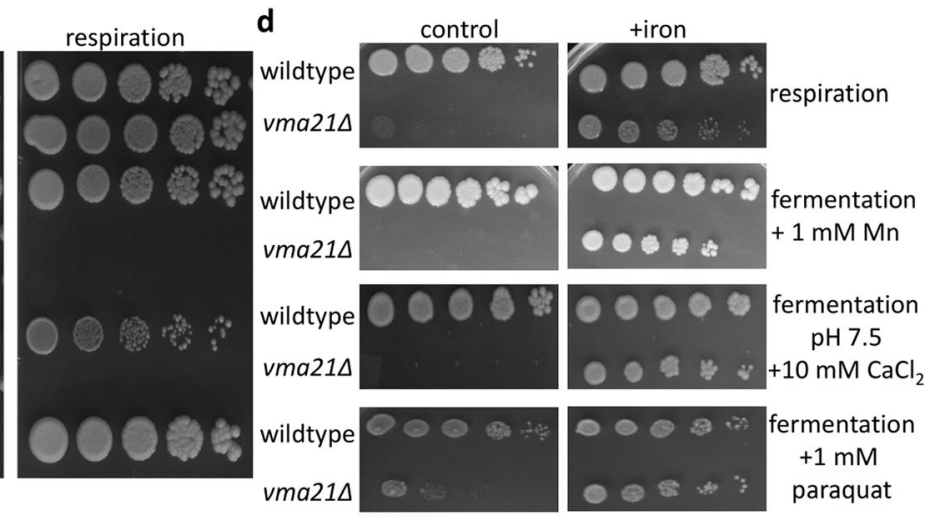

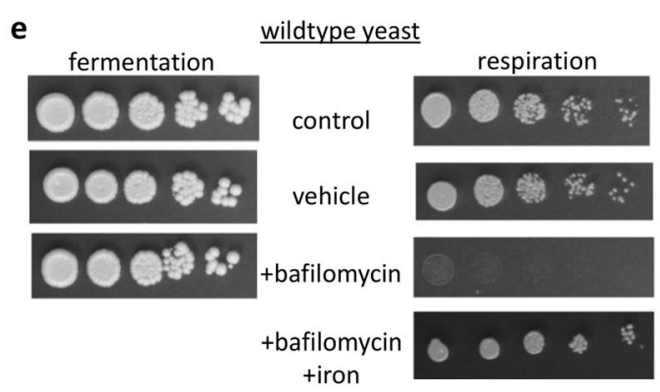

Fig. 1 Enhanced iron uptake or additional iron allows respiratory growth following impairment of the V-ATPase. a Overexpression of FET4 from a high copy $(2 \mu)$ plasmid rescues growth of vma $21 \Delta$ mutants under respiratory conditions. b A vma21 $\Delta$ mutant strain containing a suppressing loss of function mutation in the $R O X 1$ gene (rox1-A10T) rescues the vma21 $\Delta$ respiratory deficiency phenotype. c FET4 is required for $\operatorname{rox} 1 \Delta$ rescue of $v m a 21 \Delta$ respiratory growth. d Supplemental iron $(500 \mu \mathrm{M}$ ferrous ammonium sulfate) rescues pleiotropic phenotypes of vma21 $\Delta$

enzyme in the TCA cycle, and aconitase activity was reduced in vma21 $\Delta$ cells (Fig. 2d), consistent with a prior observation in vma2 mutants (Diab and Kane 2013).

Taken together, these observations suggested the possibility that disruption of iron homeostasis, and specifically, a deficiency in ISC availability, may fundamentally underlie some of the pleiotropic defects of vma mutant cells. Moreover, these observations suggest that since aging is accompanied by loss of vacuolar acidity, alterations in iron homeostasis and ISC status may occur during aging.

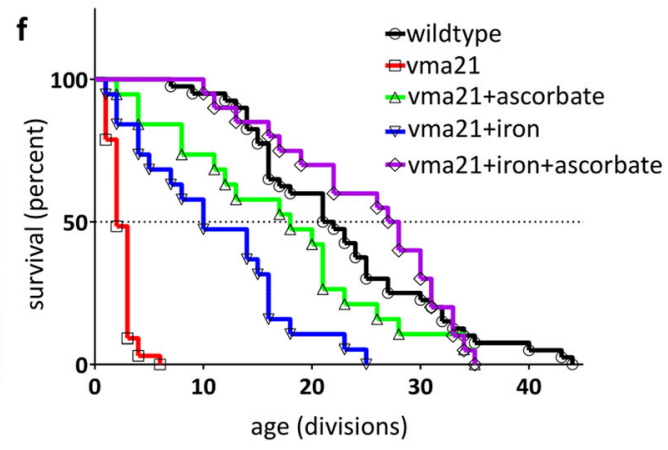

mutants. e Chemical inhibition of the V-ATPase with $3 \mu \mathrm{M}$ bafilomycin A1 preferentially impairs growth under respiratory conditions and iron rescues this phenotype. f Replicative lifespan under fermentative growth conditions (YPD media) of wild type or $v$ ma $21 \Delta$ mutants with or without $20 \mathrm{mM}$ sodium ascorbate and/or $0.5 \mathrm{mM}$ iron II sulfate. Where indicated, fermentative growth is on YPD media containing $2 \%$ glucose, and non-fermentative respiratory growth conditions is growth on YPG media containing 3\% glycerol. Lifespan statistics are shown in Table S7

Aging is characterized by a heterogeneous loss of vacuolar acidity coupled to an incompletely penetrant compensatory iron homeostatic response

To characterize the loss of vacuolar acidity during replicative aging, we tagged the vacuolar-localized carboxypeptidase Prc1 (Huh et al. 2003) with a ratiometric $\mathrm{pH}$-sensitive fluorescent protein pHluorin2 (Mahon 2011). Imaging these cells in a microfluidic device with live-cell fluorescence imaging indicated that loss of vacuolar acidity begins essentially at the start of life (Fig. 3a). By comparing different genetic strains, 

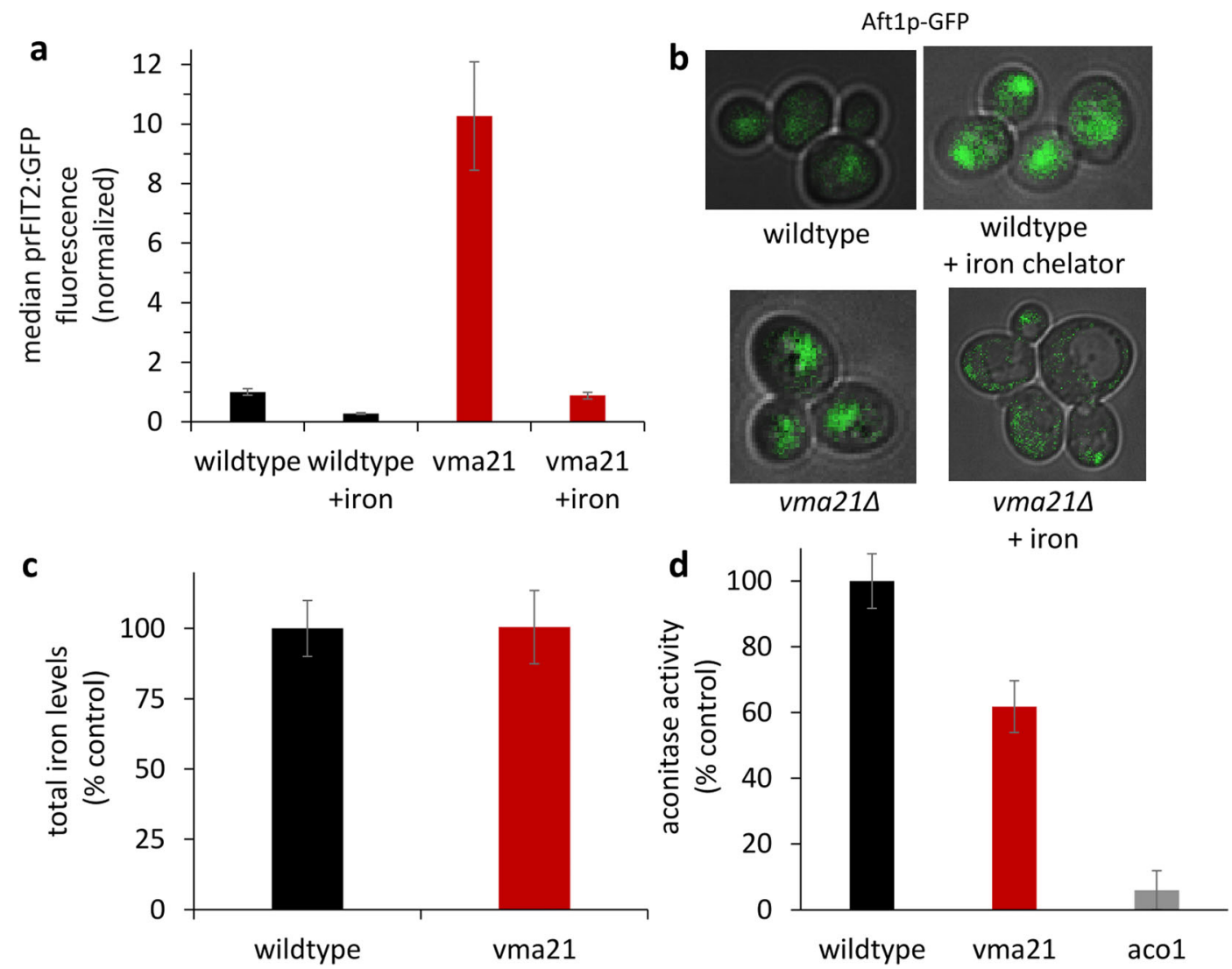

Fig. 2 Iron dyshomeostasis occurs following disruption of the VATPase. a Flow cytometry analysis of GFP levels under control of the Aft1 responsive FIT2 promoter of wild type and vma21 $\Delta$ mutants grown under fermentative conditions (YPD media) with or without $1 \mathrm{mM}$ iron. Values are the mean of three samples, each consisting of 20,000 cells per condition. Statistics are shown in Table S8. Error bars represent the standard deviation of the three sample means. b Fluorescent microscopy images of wild type or vma21 $\Delta$ mutants in the presence or absence of $100 \mu \mathrm{g} / \mathrm{ml}$

previous studies have indicated that loss of vacuolar acidity limits lifespan (Ghavidel et al. 2018; Hughes and Gottschling 2012). To test whether vacuolar acidity was a risk factor for early death in a wild-type isogenic population, we monitored changes in vacuolar acidity during early life (ages 0-12 divisions). In concordance with previous observations, the cells which experienced a faster rate of vacuolar acidity loss also exhibited a shorter lifespan (Fig. 3b, Table S4), indicating that loss of vacuolar acidity is a predictor of replicative lifespan for individual cells within an isogenic population.

Our genetic results suggested that aged cells may activate the iron regulon due to deficiency in ISCs. To test this hypothesis, we created a strain with $\mathrm{C}$ terminal fluorescent protein tags for both vacuolar

bathophenanthrolinedisulfonic acid (BPS, iron chelator) or $1 \mathrm{mM}$ iron ammonium sulfate. $\mathbf{c}$ Iron levels were measured in wild type and vma21 $\Delta$ cells grown in YPD media. No statistical difference was found using Student's $t$ test. $n=3$, error bars represent standard deviation. d Aconitase activity was measured for wild-type cells, vma21 $\Delta$, and acol $\Delta$ mutants. Each group is statistically significantly different from each other group by ANOVA and Bonferroni's multiple comparison test $P<0.001 . n=3$, error bars represent standard deviation

acidity (Prc1-pHluorin2) and an iron regulon reporter gene (Diab and Kane 2013) (Fit2-mRuby2). Averaged across the population, we observed a progressive increase in iron regulon activity during replicative aging (Fig. 3c). On a single-cell level, iron regulon activation was also associated with decreased vacuolar acidity even when controlled for age (Fig. 3d, Table S5). However, many aged cells with reduced vacuolar acidity showed little to no activation of the iron regulon (Fig. 3d). Indeed, many cells failed to activate the iron regulon to any appreciable degree during aging (Fig. 3e). Thus, population-level iron regulon activation during aging was driven by cells that induced Fit2 several orders of magnitude above cells that failed to activate the iron regulon. 
Divergent trajectories emerge during aging: active iron regulon/limited ISC deficiency and inactive iron regulon/runaway ISC deficiency

Upon observing that the age-associated iron regulon activation was only partially penetrant, we sought to evaluate the single-cell levels of ISC deficiency during aging. Is the activation of a strong iron regulon response during aging protective against ISC deficiency, or would it indicate a more severe deficiency in ISCs? To answer this question, we measured the activity of the essential ISC protein Rli1 during aging. Rli1 transports the small ribosomal subunit protein Rps2 out of the nucleus into the cytoplasm, and Rli1 activity has been used as a reporter of overall ISC sufficiency (Kispal et al. 2005). Under conditions of ISC deficiency, Rps 2 accumulates in the nucleus, so that the fraction of Rps2 localized to the nucleus is a measure of the insufficiency of active Rli1 and of ISCs generally (Kispal et al. 2005). We created a reporter strain with both an iron regulon reporter (Fit2-mRuby2) and a GFP-tagged Rps2 expressed under the GPD promoter on chromosome I. In this strain, ISC insufficiency results in the appearance of a bright fluorescent dot representing Rps2-GFP sequestered in the nucleus (Kispal et al. 2005). By observing this strain during aging in a microfluidic device, we found an overall increase in the level of Rps2-GFP foci with age, suggesting ISC insufficiency during aging (Fig. 4a). Unexpectedly, we observed that in middle-aged and old cells ( $>12$ divisions), severity of ISC deficiency was inversely correlated to the level of iron regulon activation (Fig. 4b). We assigned each cell into one of two groups-iron regulon active or iron regulon inactive-based on its maximum observed fluorescence level from Fit2mRuby 2 during aging. When we measured the overall progression of ISC deficiency during aging in the iron regulon active and inactive subpopulations, we found the ISC deficiency increased similarly for both groups until roughly division 12 . As cells entered middle age, the iron regulon-active cells plateaued in ISC deficiency, while iron reguloninactive cells became progressively more ISC deficient (Fig. 4c). Visualizing the paths of all individual cells until death reveals that iron regulon activation and ISC deficiency define two clearly divergent iron homeostasis trajectories during aging
(Fig. 4d, e): iron regulon activation/ISC sufficiency and iron regulon inactivity/ISC deficiency.

Loss of vacuolar acidity during aging is associated with elevated indications of genomic instability

Since vacuolar acidity is important for ISC sufficiency and ISC proteins mediate DNA replication and repair, the loss of vacuolar acidity may be related to genomic instability during aging. To investigate this hypothesis, we studied the development of genome instability during replicative aging in a microfluidic device using a strain with our Prc1-pH2 vacuolar acidity reporter and a mCherry-tagged Rad52, a central mediator of the DNA damage response (DDR). Upon DNA double-strand breaks, Rad52 directs the formation of a gigadaltonsized protein complex (usually one per haploid genome), which functions as processing centers for DNA repair through homologous recombination (Gasior et al. 1998). When Rad52 is fluorescently tagged, these DNA repair centers are visible as distinct subcellular foci (Lisby et al. 2001). Thus, the population frequency of Rad52 foci has been commonly used as a reporter for DNA damage severity (Alvaro et al. 2007; Novarina et al. 2017).

We and other researchers (Novarina et al. 2017) observed a population-wide increase in the frequency of activation of Rad52-mediated DNA repair during aging (Fig. 5a), indicating there may be a general increase in spontaneous DNA damage. Cells which experience spontaneous DNA damage earlier in life also had a shorter replicative lifespan (Table S6), suggesting that DNA damage may be one physiological factor that limits life. We also observed an age-controlled correlation between lower vacuolar acidity and DNA damage during aging. When looking at both activation of the DNA damage response pathway and vacuolar acidity, we observed that during middle to old age, cells in which the DNA damage response was activated had reduced vacuolar acidity compared to age-matched cells without a DNA damage response (Fig. 5b). Furthermore, a faster initial drop in vacuolar acidity during early life was correlated with an earlier appearance of DNA damage during aging (Fig. 5c). This is consistent with the idea that the ageassociated loss of vacuolar acidity is a possible upstream driver of genome instability during aging. 


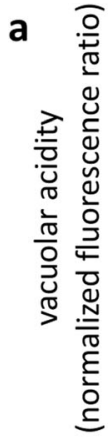

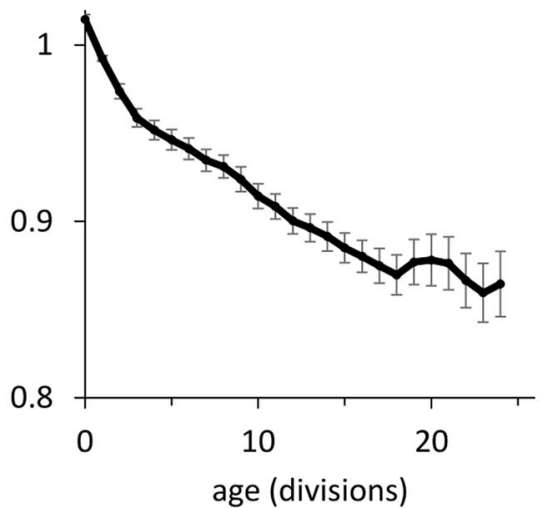

C

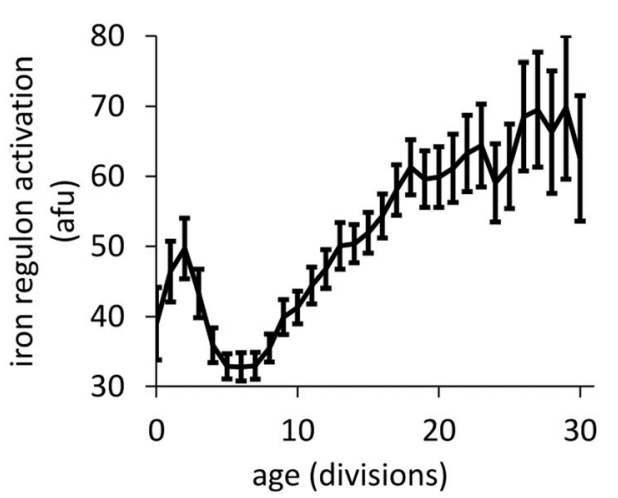

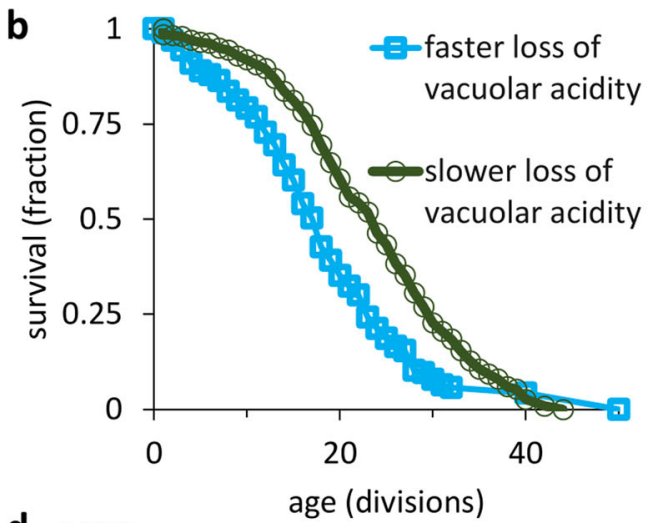

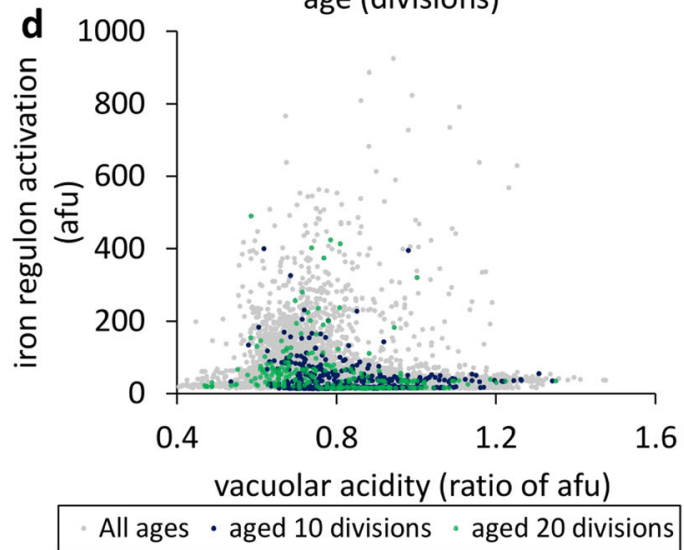

e

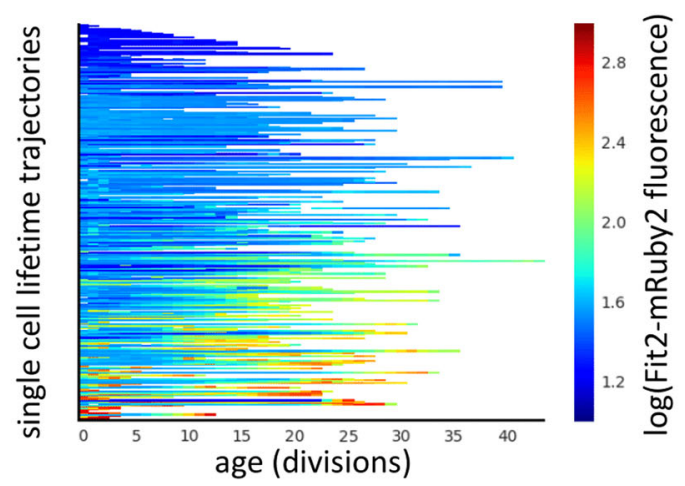

Fig. 3 Age-associated decline in vacuolar acidity is predictive of replicative lifespan and is associated with iron regulon activity which increases with age. However, a large subset of cells displays little to no iron regulon activity during aging. a Vacuolar acidity trend during aging, as measured by fluorescence ratio of vacuolelocalized Prc1-pHluorin2. Acidity is normalized per cell to young cell value (average before the second division). Pearson $r=-$ $0.259, p=1 \times 10^{-149}, n=9857$ cell divisions, error bars are standard error of the mean (SEM). b Survival curves for the population partitioned into two groups by the rate of vacuolar acidity loss during early life ( $0-12$ divisions). The rate of vacuolar acidity loss rate for each cell is calculated using the slope of the least-squares regression line through the acidity values during divisions $0-12$. Population is split in half using the median rate of vacuolar acidity loss. Fast rate of decline in vacuolar acidity is associated with shorter lifespan, logrank $p=2.22 \times 10^{-16}, n=289$ cells per group. c Age associated trend of population average iron regulon activity (Fit2-mRuby2 fluorescence). Iron regulon activity increases with age, Pearson $r=0.13, p<10^{-38}$. d Scatter plot of iron regulon activity (Fit2-mRuby2 fluorescence) and vacuolar acidity for all cells at all ages. Iron regulon activity is correlated with lower vacuolar acidity even when controlled for age (all ages: Spearman $\rho=-0.21, p<10^{-4}$; age 10: Spearman $\rho=-0.17, p=0.0017$; age 20: Spearman $\left.\rho=-0.38, p<10^{-4}\right)$. e Single-cell lifetime trajectories of measured iron regulon activity. Color values are log (Fit2mRuby2 fluorescence). Many cells show limited to no iron regulon activation 

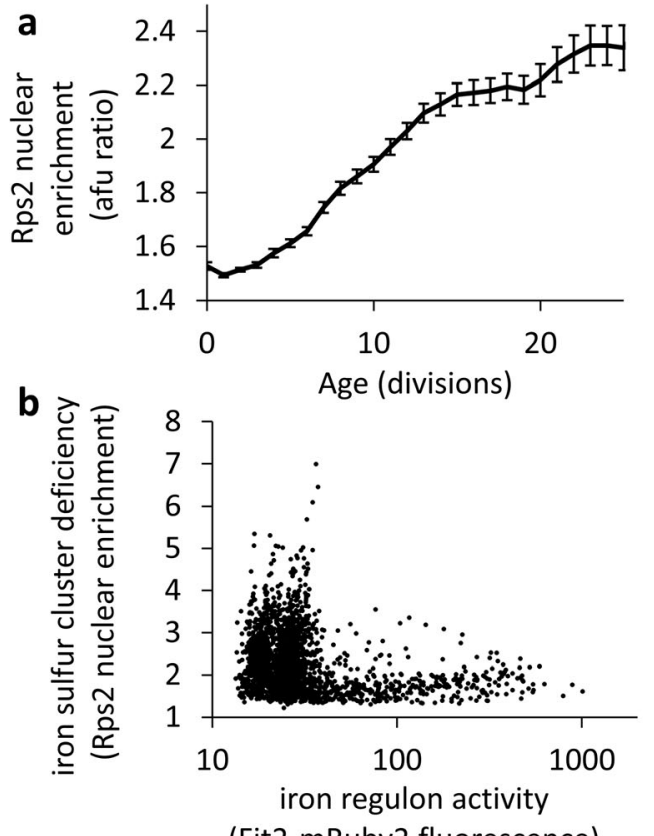

(Fit2-mRuby2 fluorescence)

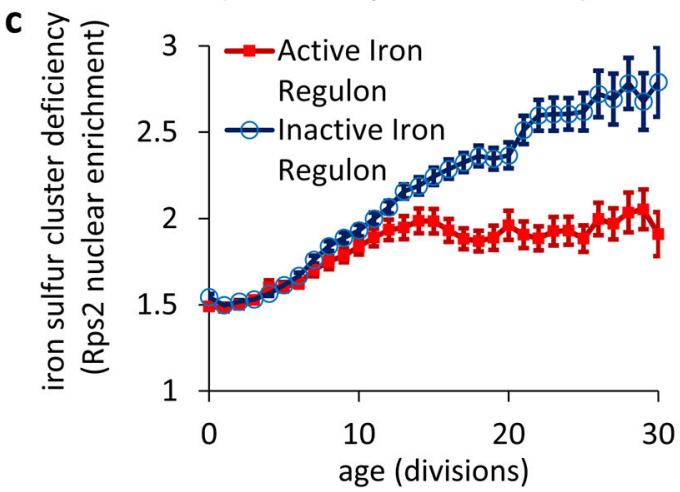

Fig. 4 Iron-sulfur clusters become deficient during aging. Activation of iron regulon is inversely correlated with iron-sulfur cluster deficiency. Cells with robust iron regulon activity during aging generally follow a trajectory of limited iron-sulfur cluster deficiency. $n=209$ total cells. a Aging trend of population mean iron-sulfur cluster insufficiency (Rps2 nuclear enrichment). Iron-sulfur cluster insufficiency increases during aging. Pearson $r=0.52, p<10^{-4}$. Error bars are SEM. b Scatter plot of iron-sulfur cluster insufficiency (nuclear enrichment of Rps2-GFP) and iron regulon activation (Fit2-mRuby2 fluorescence) in aged cells (all cells and ages $>12$ divisions). Spearman $\rho=-0.26, p<10^{-4}, n=189$ (cells that lived 12 generations or more). c Aging trend of iron-sulfur cluster deficiency for iron regulon competent and incompetent cells during aging. Iron regulon-active cells are defined as having a maximum Fit2 fluorescence level that is

The iron homeostasis trajectory of a cell is associated with its genome stability

Iron metabolism and genome maintenance are interconnected, as ISCs are necessary for both DNA replication

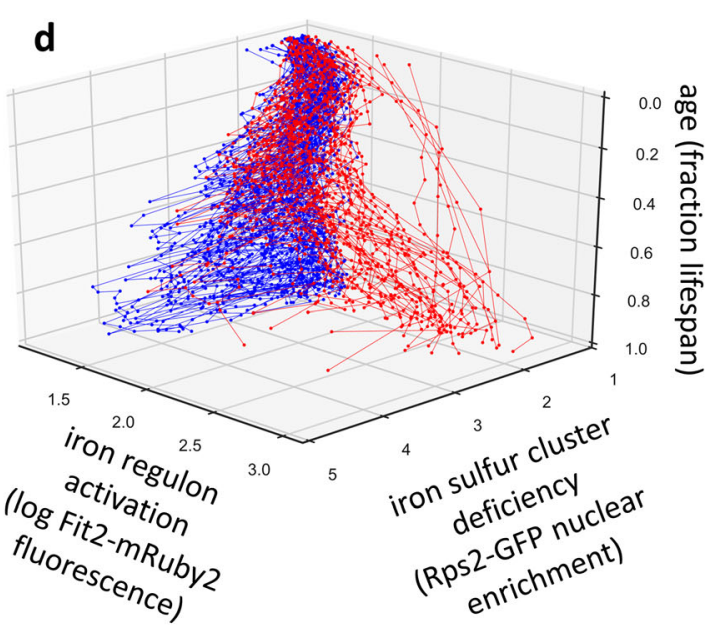

e

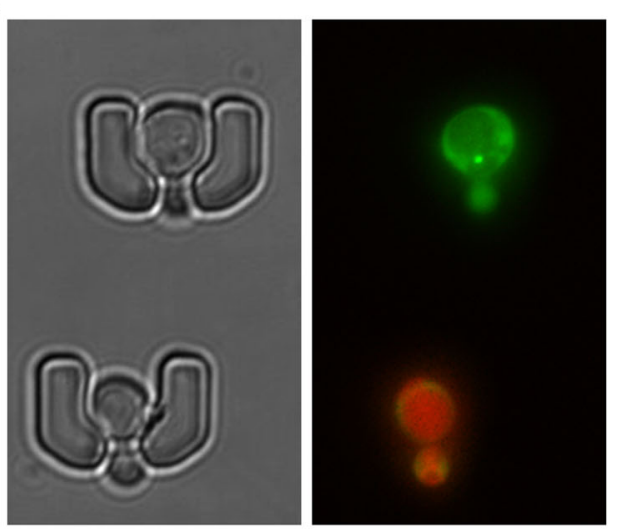

$3 \times$ the median baseline. Iron regulon-inactive cells are not more ironstarved during early life, but have less severe iron-sulfur cluster deficiency from middle to old age $(p<0.05$ for ages $15-30)$. $n_{\text {active }}$ iron regulon $=59, n_{\text {inactive iron regulon }}=150$. Error bars are SEM. $\mathbf{d}$ Singlecell trajectories of iron-sulfur cluster deficiency and iron regulon activity during aging. Cells display divergent iron metabolism trajectories during aging. Red: iron regulon active with limited iron-sulfur cluster deficiency. Blue: no iron regulon activation. $n=209$. e Example aged cells in adjacent traps from each iron metabolism trajectory. Top cell in trap: iron-sulfur cluster deficiency (Rps2-GFP nuclear retention visible as bright green dot) with little iron regulon activation. Bottom cell in trap: robust iron regulon activation (Fit2-mRuby2) with no visible Rps2-GFP nuclear retention

and many types of DNA repair (Netz et al. 2014). Accordingly, impairment of ISC production has been shown to cause genome instability (Veatch et al. 2009) and reduced survival in genotoxic conditions (Pijuan et al. 2015). Because of this, differences in genome 
stability between the two subpopulations of divergent iron metabolism aging trajectories were evaluated. Strains were generated with both an iron regulon reporter (Fit2-mRuby2) and a DNA damage reporter (Rad52GFP) and replicative aging was measured in a microfluidic device. Cells were separated into two groups, either iron regulon active or iron regulon inactive based on the maximum iron regulon activity observed during aging. Cells which activated the iron regulon during aging survived for longer after the initial observation of spontaneous DNA damage (Fig. 6a, b). Iron regulon-active cells also underwent more cell divisions during which the DNA damage response had been activated (Fig. 6c). We speculate that because the iron regulon-active cells experienced limited ISC deficiency during aging, these cells may be better able to survive spontaneous age-related DNA damage by marshalling the appropriate ISC-dependent DNA repair processes.

Interestingly, differing relationships between DNA damage response activation and longevity between these two iron metabolism subpopulations were observed. Within the iron regulon-active group, longer lifespan was correlated with additional divisions during which the DNA damage response was activated (Fig. 6d). Within the iron regulon-inactive group, there was no correlation between longer lifespan and activation of the DNA damage response (Fig. 6d). One explanation of these results is that the longest-lived cells within these groups may have different molecular processes underlying their survival. Within the iron regulon-active group, longevity may be determined by the robustness of the repair and survival responses to spontaneous DNA damage. Within the iron reguloninactive group, with more severe ISC deficiency and potentially less capability to repair DNA damage, longevity may be more dependent on lower rates of spontaneous DNA damage during aging.

\section{Discussion}

Using a combination of traditional yeast genetics, microfluidics, and fluorescence microscopy, we have further characterized the cellular consequences of loss of vacuolar acidity, an early life physiological change during aging. We found that loss of vacuolar acidity results in iron-dependent pleiotropic phenotypes of vma mutant cells. This suggests that iron dyshomeostasis is an important consequence following disruption of the V-ATPase. In
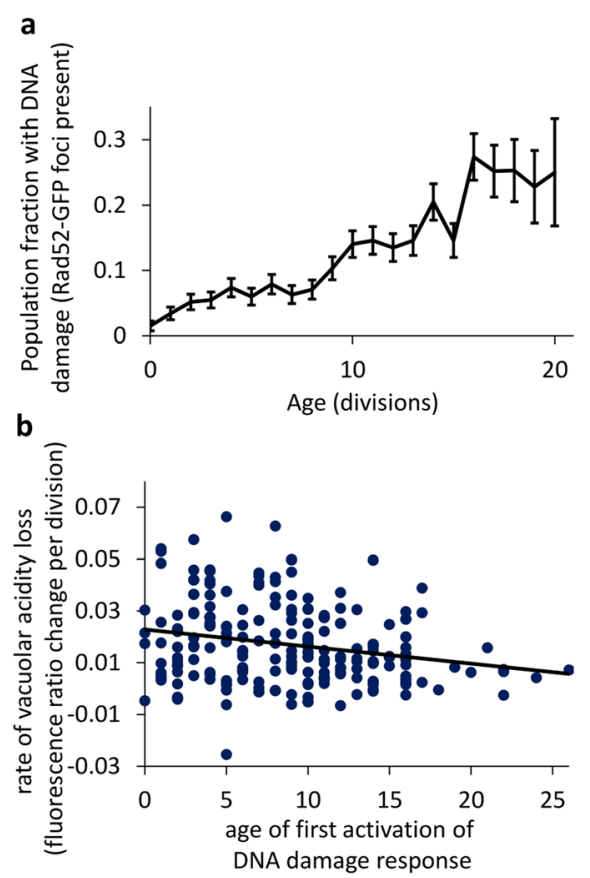

C

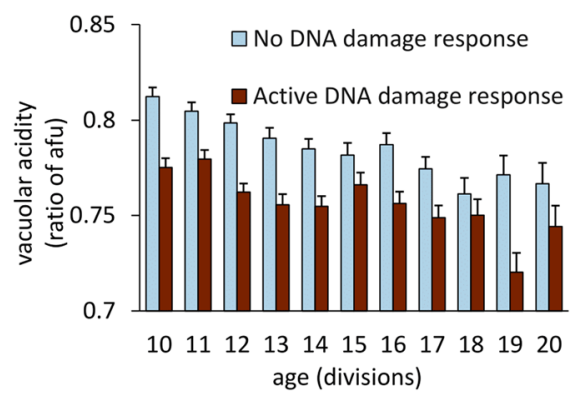

Fig. 5 Activation of the DNA damage response increases during aging, is correlated with the loss of vacuolar acidity, and is predictive of a shorter replicative lifespan. a Aging trend of DNA damage response activation as measured by presence of Rad52-GFP foci. As cells age, the fraction of cells that activate the DNA damage response increases, Pearson $r=0.27, p<10^{-4}, n=266$ cells. Error bars are SEM. b Scatter plot of age of first DNA damage response activation (Rad52-GFP foci) and rate of vacuolar acidity loss in early life for cells which activate the DNA damage response at any point during life (ages $0-12$ divisions). $n=244$ cells that developed foci. Faster loss of vacuolar acidity during early life (ages $1-12$ divisions) correlates with earlier appearance of DNA damage response. Pearson $r=-0.23, p=$ 0.0006. c Comparison of vacuolar acidity for cells with and without activated DNA damage response (Rad52-GFP foci) at various ages. $n=266$. Presence of activated DNA damage response (Rad52-GFP foci) is associated with lower vacuolar acidity when controlled for age. All comparisons ranksum $p<0.05$ except for ages 15: $p=0.089,18$ : $p=0.45$, and 20: $p=0.20$. Error bars are SEM

vma mutants, there is both a loss of vacuolar acidity as well as cytosolic acidification (Martinez-Munoz and Kane 2008). Intracellular acidification can damage iron-sulfur 
a

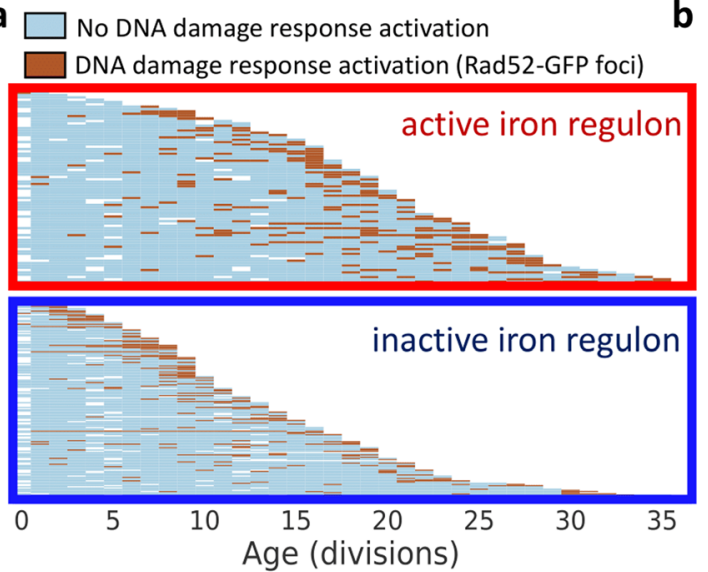

b

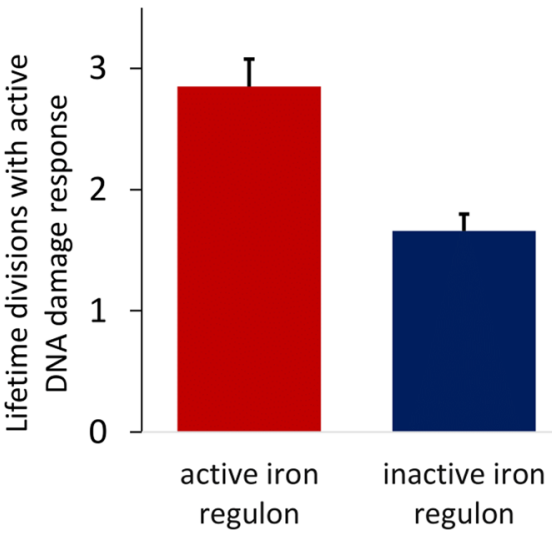

C

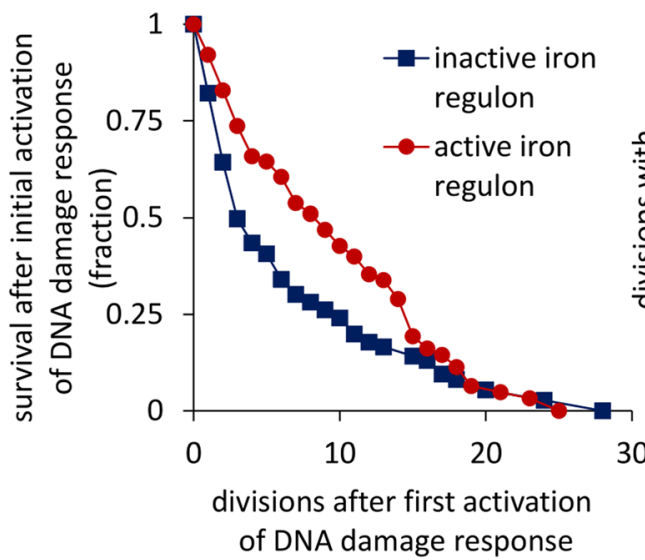

Fig. 6 Iron regulon-active cells survive longer after the first activation of the DNA damage response during aging and undergo more lifetime divisions during which the DNA damage response (Rad52-GFP foci formation) is activated. Total lifespan of iron regulon-active cells is highly correlated to the number of lifetime divisions during which the DNA damage response is activated. Within the iron regulon-inactive group, longer-lived cells do not undergo any more divisions with DNA damage than shorter lived cells. This suggests that within the iron regulon competent subpopulation, the longest-lived cells are those with the most robust ability to survive age-associated DNA damage. Within the iron regulon incompetent subpopulation, the longest-lived cells are those with minimal age-associated DNA damage. a Single-cell trajectories of DNA damage response activation during aging. Top: cells that activate the iron regulon during aging (maximum lifetime Fit2-mCherry fluorescence $>3 \times$ median baseline level). Bottom: cells that fail to activate the iron regulon during aging. Empty spaces indicate that data was not collected for a single cell at that age, e.g., due to temporary cell segmentation or tracking

clusters (Follmann et al. 2009; Li et al. 2011), and cytosolic acidification by acetic acid treatment is sufficient to activate the iron regulon (Diab and Kane 2013). This indicates that cytosolic acidification following loss of the V-ATPase may contribute to activating the iron regulon by damaging

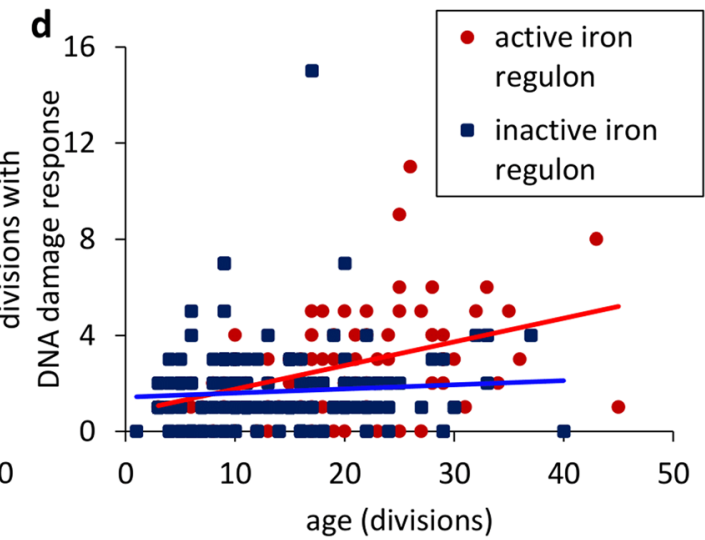

error or initial age of capture after first division. b Survival curves comparing remaining lifespan after first observed activation of the DNA damage response (Rad52-GFP foci formation) for iron regulon competent and incompetent cells. Cells that activate the iron regulon during aging have increased survival after the first activation of the DNA damage response logrank $p=0.018, n_{\text {active }}$ iron regulon $=76, n_{\text {inactive iron regulon }}=129$. Error bars are SEM. $\mathbf{c}$ Comparison of DNA damage response activation between iron regulon active and iron regulon-inactive cells. During aging, cells that activate the iron regulon undergo more divisions during which the DNA damage response is activated (Student's $t p<10^{-4}$ ). d Scatter plots showing correlation between total lifespan and number of divisions during which the DNA damage response is activated. Longer lifespan of the iron regulon-active cells is wellcorrelated (Pearson $r=0.41$ ) with a higher number of divisions with an activated DNA damage response. Longer lifespan in iron regulon-inactive cells is not correlated (Pearson $r=0.08$ ) with more divisions with DNA damage. Correlation coefficients are significantly different by Fisher's Z-transform $p=0.01$

iron-sulfur clusters. With age, $\mathrm{pH}$ near the cytoplasmic membrane has been reported to increase (Henderson et al. 2014), while cytosolic $\mathrm{pH}$ has been reported to acidify (Knieß and Mayer 2016). It is possible that during aging, cytosolic acidification may result in damage to iron- 
sulfur clusters. Yeast V-ATPase mutants display elevated levels of intracellular reactive oxygen species (ROS) (Milgrom et al. 2007) and ISCs are highly sensitive to degradation by ROS (Jang and Imlay 2007). Interestingly, disruption of ISC production is itself also a source of ROS (Gomez et al. 2014), suggesting the possibility of vicious cycle during aging.

What specifically happens to iron homeostasis following alterations in cellular $\mathrm{pH}$ homeostasis remains to be determined. Yeast V-ATPase mutants exhibit defects in the maturation of Fet3, a high affinity iron uptake protein (Davis-Kaplan et al. 2004). However, fet3 mutants do not fully phenocopy vma mutants, and we found no difference in total cellular iron levels in vma21 cells, suggesting additional factors beyond reduced iron uptake lead to the pleiotropic phenotypes.

In rodent cultured hepatocytes, inhibition of the V-ATPase results in the release of chelatable iron from lysosomes, which is then taken up by mitochondria (Uchiyama et al. 2008). In aging mice, iron has also been found to accumulate in mitochondria (Seo et al. 2008). Disruption of mitochondrial ironsulfur cluster biosynthesis in yeast results in the accumulation of iron within the mitochondria and activation of the iron regulon in spite of normal cytosolic iron levels (Chen et al. 2004). The iron regulon activation and iron levels we and others observe in vma mutants are consistent with these observations. However, since we observe that additional iron rescues a mitochondrial defect in vma mutants, a model of mitochondrial iron accumulation would seem to require that the mitochondria would harbor iron in a form that is not bioavailable. Future studies could assess the intracellular localization, trafficking, and redox state of iron in vma mutants in order to identify if iron is mislocalized and/or if the redox state of iron is aberrant, and these results could then generate hypotheses for what may occur following age-associated $\mathrm{pH}$ dyshomeostasis.

We found that wild-type aged yeast cells display a deficiency in iron-sulfur clusters and an increased DNA damage response. Furthermore, both of these physiological declines correlate with vacuolar acidity loss when controlled for age. Unexpectedly, we found that only a subset of yeast cells can activate the expected iron regulon program in response to iron-sulfur cluster deficiency during aging. Thus, an isogenic, environmentally homogeneous population of yeast cells follows divergent iron metabolism trajectories during aging. Iron regulon-active cells mount a robust activation of the iron regulon, resulting in limited ISC deficiency throughout life. In contrast, more than half of the population is unable to mount any meaningful iron regulon activity, resulting in a progressive worsening of ISC deficiency during aging. This divergence has implications on other measures of physiology during aging, including cellular responses to age-associated spontaneous DNA damage. We find that in general, iron regulon-active cells are better able to survive and continue dividing during episodes of apparent DNA damage, with a strong correlation between longer lifespan and additional DNA damage episodes survived. In contrast, iron reguloninactive cells appear sensitive to DNA damage, and longer-lived cells in this population appear to be those which generally escaped DNA damage early in life, requiring minimal activation of the DNA damage response. We have not attempted to fully characterize the differences between the iron regulon-active and iron regulon-inactive subpopulations, including the mechanistic details of DNA damage survival during aging. While the fidelity of DNA repair was not investigated, we observed an intriguing divergence during aging of single cells into a DNA damage-resistant and vulnerable states underpinned by the activation of (or lack thereof) the iron regulon gene expression program.

The concept of cell-state divergence during aging that we describe has recently been characterized in terms of yeast mother and daughter cell morphology (Jin et al. 2019). Although no gene expression or mechanistic observations were made in that study, our shared observations suggest that the aging process may have characteristics reminiscent of Waddington's landscape of development, where cells diverge into distinct aged states. The mechanistic underpinnings of these apparently stochastic paths of aging present a novel and intriguing area of study. We expect that aging trajectories may be more fully characterized by single-cell observation of additional gene expression or physiological parameters. While this study and Jin et al. (2019) made observations of different parameters, it seems likely that integration of these observations as well as those of other physiological variables will result in the identification of more divergent physiological variables during aging. Ultimately, whether the total multidimensional landscape of aging can be best understood as a finite array of valleys, or whether this analogy is better suited to a separate understanding of single parameters of aging is yet to be determined. 
The loss of vacuolar acidity is an evolutionarily conserved aging phenotype identified thus far in yeast (Ghavidel et al. 2018; Hughes and Gottschling 2012) and worms (Baxi et al. 2017). Future studies will need to directly assess whether loss of lysosomal acidity occurs in mammalian cell types with age or age-associated diseases, which could be testable using ratiometric $\mathrm{pH}$ sensors. If loss of lysosomal acidity occurs during mammalian aging, further studies could seek to determine if it is a driving force for age-associated iron accumulation, mitochondrial dysfunction, and/or ISC dysfunction. Impairment of lysosomal acidity has been tied to iron dyshomeostasis in mammalian cell lines (Lim et al. 2007; Miles et al. 2017; Schneider et al. 2015; Straud et al. 2010). ISC proteins function in a wide variety of physiological contexts and are highly conserved evolutionarily (Netz et al. 2014). Moreover, defects in ISC production or in specific ISC proteins underlie numerous genetic syndromes with progeroid characteristics as well as many age-associated diseases (Perera and Zoncu 2016). We propose that a $\mathrm{pH}$-dependent impairment of iron-sulfur clusters with age may be a fundamental and evolutionarily conserved driver of clinically significant age-associated pathology.

Funding information KLC was funded by National Institute of Aging F30AG052225. MMC was supported by NIH Training Grant T32AG000057. AJH acknowledges funding (R01GM118854) from the National Institute of General Medical Sciences (NIGMS). SRK was funded by U.S. Department of Defense DOD/CDMRP grant W81XWH-16-1-0579. MK was supported by National Institute of Aging grants P30AG013280 and R01AG056359. BMW was funded by University of HoustonClear Lake Faculty Research Support Funds (Award Number A2S19).

\section{References}

Alberti S, Gitler AD, Lindquist S (2007) A suite of Gateway® cloning vectors for high-throughput genetic analysis in Saccharomyces cerevisiae. Yeast Chichester Engl 24:913919

Alvaro D, Lisby M, Rothstein R (2007) Genome-wide analysis of Rad52 foci reveals diverse mechanisms impacting recombination. PLoS Genet 3:e228

Bakker E, Swain PS, Crane MM (2018) Morphologically constrained and data informed cell segmentation of budding yeast. Bioinformatics 34:88-96

Baxi K, Ghavidel A, Waddell B, Harkness TA, de Carvalho CE (2017) Regulation of lysosomal function by the DAF-16 forkhead transcription factor couples reproduction to aging in Caenorhabditis elegans. Genetics 207:83-101

Birkeland SR, Jin N, Ozdemir AC, Lyons RH, Weisman LS, Wilson TE (2010) Discovery of mutations in Saccharomyces cerevisiae by pooled linkage analysis and whole-genome sequencing. Genetics 186:1127-1137

Carmona-Gutierrez D, Hughes AL, Madeo F, Ruckenstuhl C (2016) The crucial impact of lysosomes in aging and longevity. Ageing Res Rev 32:2-12

Chen OS, Crisp RJ, Valachovic M, Bard M, Winge DR, Kaplan J (2004) Transcription of the yeast iron regulon does not respond directly to iron but rather to iron-sulfur cluster biosynthesis. J Biol Chem 279:29513-29518

Chen KL, Crane MM, Kaeberlein M (2017) Microfluidic technologies for yeast replicative lifespan studies. Mech Ageing Dev 161:262-269

Chen KL, Ven TN, Crane MM, Chen DE, Feng YC, Suzuki N, Russell AE, de Moraes D, Kaeberlein M (2019) An inexpensive microscopy system for microfluidic studies in budding yeast. Translational Medicine of Aging 3:52-56

Crane MM, Kaeberlein M (2018) The paths of mortality: how understanding the biology of aging can help explain systems behavior of single cells. Curr Opin Syst Biol 8:25-31

Crane MM, Clark IBN, Bakker E, Smith S, Swain PS (2014) A microfluidic system for studying ageing and dynamic singlecell responses in budding yeast. PLoS One 9:e100042

Davis-Kaplan SR, Ward DM, Shiflett SL, Kaplan J (2004) Genome-wide analysis of iron-dependent growth reveals a novel yeast gene required for vacuolar acidification. J Biol Chem 279:4322-4329

Díaz de la Loza MDC, Gallardo M, García-Rubio ML, Izquierdo A, Herrero E, Aguilera A, Wellinger RE (2011) Zim17/ Tim15 links mitochondrial iron-sulfur cluster biosynthesis to nuclear genome stability. Nucleic Acids Res 39:60026015

de Silva D, Davis-Kaplan S, Fergestad J, Kaplan J (1997) Purification and characterization of Fet3 protein, a yeast homologue of ceruloplasmin. J Biol Chem 272:1420814213

Diab HI, Kane PM (2013) Loss of vacuolar H+-ATPase (VATPase) activity in yeast generates an iron deprivation signal that is moderated by induction of the peroxiredoxin TSA2. J Biol Chem 288:11366-11377

Eide DJ, Bridgham JT, Zhao Z, Mattoon JR (1993) The vacuolar $\mathrm{H}(+)$-ATPase of Saccharomyces cerevisiae is required for efficient copper detoxification, mitochondrial function, and iron metabolism. Mol Gen Genet 241:447-456

Follmann M, Ochrombel I, Kramer R, Trotschel C, Poetsch A, Ruckert C, Huser A, Persicke M, Seiferling D, Kalinowski J et al (2009) Functional genomics of pH homeostasis in Corynebacterium glutamicum revealed novel links between $\mathrm{pH}$ response, oxidative stress, iron homeostasis and methionine synthesis. BMC Genomics 10:621

Gasior SL, Wong AK, Kora Y, Shinohara A, Bishop DK (1998) Rad52 associates with RPA and functions with $\operatorname{Rad} 55$ and Rad57 to assemble meiotic recombination complexes. Genes Dev 12:2208-2221

Ghavidel A, Baxi K, Prusinkiewicz M, Swan C, Belak ZR, Eskiw CH, Carvalho CE, Harkness TA (2018) Rapid nuclear exclusion of Hcml in aging Saccharomyces cerevisiae leads to 
vacuolar alkalization and replicative senescence. G3 Genes Genomes Genet 8:1579-1592

Gomez M, Perez-Gallardo RV, Sanchez LA, Diaz-Perez AL, Cortes-Rojo C, Meza Carmen V, Saavedra-Molina A, LaraRomero J, Jimenez-Sandoval S, Rodriguez F et al (2014) Malfunctioning of the iron-sulfur cluster assembly machinery in Saccharomyces cerevisiae produces oxidative stress via an iron-dependent mechanism, causing dysfunction in respiratory complexes. PLoS One 9:e111585

Henderson KA, Hughes AL, Gottschling DE (2014) Motherdaughter asymmetry of $\mathrm{pH}$ underlies aging and rejuvenation in yeast. Elife 3:e03504

Hughes AL, Gottschling DE (2012) An early age increase in vacuolar $\mathrm{pH}$ limits mitochondrial function and lifespan in yeast. Nature 492:261-265

Huh W-K, Falvo JV, Gerke LC, Carroll AS, Howson RW, Weissman JS, O'Shea EK (2003) Global analysis of protein localization in budding yeast. Nature 425:686-691

Inoue H, Noumi T, Nagata M, Murakami H, Kanazawa H (1999) Targeted disruption of the gene encoding the proteolipid subunit of mouse vacuolar H+-ATPase leads to early embryonic lethality. Biochim Biophys Acta 1413:130-138

Jang S, Imlay JA (2007) Micromolar intracellular hydrogen peroxide disrupts metabolism by damaging iron-sulfur enzymes. J Biol Chem 282:929-937

Jensen LT, Culotta VC (2002) Regulation of Saccharomyces cerevisiae FET4 by oxygen and iron. J Mol Biol 318:251260

Jin M, Li Y, O'Laughlin R, Bittihn P, Pillus L, Tsimring LS, Hasty J, Hao N (2019) Divergent aging of isogenic yeast cells revealed through single-cell phenotypic dynamics. Cell Syst 8:242-253.e3

Kidane TZ, Sauble E, Linder MC (2006) Release of iron from ferritin requires lysosomal activity. Am J Physiol Cell Physiol 291:C445-C455

Kispal G, Sipos K, Lange H, Fekete Z, Bedekovics T, Janáky T, Bassler J, Aguilar Netz DJ, Balk J, Rotte C, Lill R (2005) Biogenesis of cytosolic ribosomes requires the essential ironsulphur protein Rli1p and mitochondria. EMBO J 24:589598

Knieß RA, Mayer MP (2016) The oxidation state of the cytoplasmic glutathione redox system does not correlate with replicative lifespan in yeast. Npj Aging Mech Dis 2:1-11

Lee MB, Dowsett IT, Carr DT, Wasko BM, Stanton SG, Chung MS, Ghodsian N, Bode A, Kiflezghi MG, Uppal PA et al (2019) Defining the impact of mutation accumulation on replicative lifespan in yeast using cancer-associated mutator phenotypes. Proc Natl Acad Sci U A 116:3062-3071

Li SC, Kane PM (2009) The yeast lysosome-like vacuole: endpoint and crossroads. Biochim Biophys Acta 1793:650-663

Li H, Mapolelo DT, Dingra NN, Keller G, Riggs-Gelasco PJ, Winge DR, Johnson MK, Outten CE (2011) Histidine 103 in Fra2 is an iron-sulfur cluster ligand in the [2Fe-2S] Fra2Grx 3 complex and is required for in vivo iron signaling in yeast. J Biol Chem 286:867-876

Liao C, Hu B, Arno MJ, Panaretou B (2007) Genomic screening in vivo reveals the role played by vacuolar $\mathrm{H}+$ ATPase and cytosolic acidification in sensitivity to DNA-damaging agents such as cisplatin. Mol Pharmacol 71:416-425

Lill R, Hoffmann B, Molik S, Pierik AJ, Rietzschel N, Stehling O, Uzarska MA, Webert H, Wilbrecht C, Mühlenhoff U (2012)
The role of mitochondria in cellular iron-sulfur protein biogenesis and iron metabolism. Biochim. Biophys. Acta BBA Mol. Cell Res. 1823:1491-1508

Lim J-H, Park J-W, Kim SJ, Kim M-S, Park S-K, Johnson RS, Chun Y-S (2007) ATP6V0C competes with von HippelLindau protein in hypoxia-inducible factor 1alpha (HIF1alpha) binding and mediates HIF-1alpha expression by bafilomycin A1. Mol Pharmacol 71:942-948

Lisby M, Rothstein R, Mortensen UH (2001) Rad52 forms DNA repair and recombination centers during $\mathrm{S}$ phase. Proc Natl Acad Sci 98:8276-8282

López-Otín C, Blasco MA, Partridge L, Serrano M, Kroemer G (2013) The hallmarks of aging. Cell 153:1194-1217

Mahon MJ (2011) pHluorin2: an enhanced, ratiometric, pHsensitive green florescent protein. Adv Biosci Biotechnol 2: $132-137$

Martinez-Munoz GA, Kane P (2008) Vacuolar and plasma membrane proton pumps collaborate to achieve cytosolic $\mathrm{pH}$ homeostasis in yeast. J Biol Chem 283:20309-20319

McCormick MA, Delaney JR, Tsuchiya M, Tsuchiyama S, Shemorry A, Sim S, Chou AC-Z, Ahmed U, Carr D, Murakami CJ et al (2015) A comprehensive analysis of replicative lifespan in 4,698 single-gene deletion strains uncovers conserved mechanisms of aging. Cell Metab 22:895906

Miles AL, Burr SP, Grice GL, Nathan JA (2017) The vacuolarATPase complex and assembly factors, TMEM199 and CCDC115, control HIF1 $\alpha$ prolyl hydroxylation by regulating cellular iron levels. eLife 6:e22693

Milgrom E, Diab H, Middleton F, Kane PM (2007) Loss of vacuolar proton-translocating ATPase activity in yeast results in chronic oxidative stress. J Biol Chem 282:7125-7136

Nasmyth KA, Reed SI (1980) Isolation of genes by complementation in yeast: molecular cloning of a cell-cycle gene. Proc Natl Acad Sci 77:2119-2123

Netz DJA, Mascarenhas J, Stehling O, Pierik AJ, Lill R (2014) Maturation of cytosolic and nuclear iron-sulfur proteins. Trends Cell Biol 24:303-312

Novarina D, Mavrova SN, Janssens GE, Rempel IL, Veenhoff LM, Chang M (2017) Increased genome instability is not accompanied by sensitivity to DNA damaging agents in aged yeast cells. DNA Repair 54:1-7

Ohya Y, Ohsumi Y, Anraku Y (1986) Isolation and characterization of Ca2+-sensitive mutants of Saccharomyces cerevisiae. J Gen Microbiol 132:979-988

Perera RM, Zoncu R (2016) The lysosome as a regulatory hub. Annu Rev Cell Dev Biol 32:223-253

Philpott CC, Smith PM (2013). Iron Starvation Response in Saccharomyces cerevisiae. In Encyclopedia of Inorganic and Bioinorganic Chemistry, R.A. Scott (Ed.)

Pijuan J, Maria C, Herrero E, Belli G (2015) Impaired mitochondrial $\mathrm{Fe}-\mathrm{S}$ cluster biogenesis activates the DNA damage response through different signaling mediators. J Cell Sci 128:4653-4665

Rutherford JC, Ojeda L, Balk J, Mühlenhoff U, Lill R, Winge DR (2005) Activation of the iron regulon by the yeast Aft1/Aft2 transcription factors depends on mitochondrial but not cytosolic iron-sulfur protein biogenesis. J Biol Chem 280:1013510140 
Sasikumar AN, Killilea DW, Kennedy BK, Brem RB (2019) Potassium restriction boosts vacuolar acidity and extends lifespan in yeast. Exp Gerontol 120:101-106

Schleit J, Johnson SC, Bennett CF, Simko M, Trongtham N, Castanza A, Hsieh EJ, Moller RM, Wasko BM, Delaney JR et al (2013) Molecular mechanisms underlying genotypedependent responses to dietary restriction. Aging Cell 12: 1050-1061

Schneider LS, von Schwarzenberg K, Lehr T, Ulrich M, KubischDohmen R, Liebl J, Trauner D, Menche D, Vollmar AM (2015) Vacuolar-ATPase inhibition blocks Iron metabolism to mediate therapeutic effects in breast cancer. Cancer Res 75: 2863-2874

Seo AY, Xu J, Servais S, Hofer T, Marzetti E, Wohlgemuth SE, Knutson MD, Chung HY, Leeuwenburgh C (2008) Mitochondrial iron accumulation with age and functional consequences. Aging Cell 7:706-716

Serrano R, Bernal D, Simon E, Arino J (2004) Copper and iron are the limiting factors for growth of the yeast Saccharomyces cerevisiae in an alkaline environment. J Biol Chem 279: 19698-19704

Straud S, Zubovych I, De Brabander JK, Roth MG (2010) Inhibition of iron uptake is responsible for differential sensitivity to V-ATPase inhibitors in several cancer cell lines. PLoS One 5:e11629

Szczypka MS, Zhu Z, Silar P, Thiele DJ (1997) Saccharomyces cerevisiae mutants altered in vacuole function are defective in copper detoxification and iron-responsive gene transcription. Yeast Chichester Engl. 13:1423-1435
Ter Linde JJ, Steensma HY (2002) A microarray-assisted screen for potential Hap1 and Rox1 target genes in Saccharomyces cerevisiae. Yeast 19:825-840

Tong W-H, Rouault TA (2006) Functions of mitochondrial ISCU and cytosolic ISCU in mammalian iron-sulfur cluster biogenesis and iron homeostasis. Cell Metab 3:199-210

Uchiyama A, Kim J-S, Kon K, Jaeschke H, Ikejima K, Watanabe S, Lemasters JJ (2008) Translocation of iron from lysosomes into mitochondria is a key event during oxidative stressinduced hepatocellular injury. Hepatol Baltim Md 48:1644 1654

Veatch JR, McMurray MA, Nelson ZW, Gottschling DE (2009) Mitochondrial dysfunction leads to nuclear genome instability via an iron-sulfur cluster defect. Cell 137:1247-1258

Wasko BM, Holland CL, Resnick MA, Lewis LK (2009) Inhibition of DNA double-strand break repair by the $\mathrm{Ku}$ heterodimer in mrx mutants of Saccharomyces cerevisiae. DNA Repair 8:162-169

Wasko BM, Carr DT, Tung H, Doan H, Schurman N, Neault JR, Feng J, Lee J, Zipkin B, Mouser J et al (2013) Buffering the $\mathrm{pH}$ of the culture medium does not extend yeast replicative lifespan. F1000Res 2:216

Waters BM, Eide DJ (2002) Combinatorial control of yeast FET4 gene expression by iron, zinc, and oxygen. J Biol Chem 277: 33749-33757

Publisher's note Springer Nature remains neutral with regard to jurisdictional claims in published maps and institutional affiliations. 